\title{
Applying e-learning for Multicultural Healthcare Education
}

\author{
Myonghwa Park ${ }^{1}$ and Moonhee Gang ${ }^{1 *}$ \\ ${ }^{1}$ College of Nursing, Chungnam National University, \\ Daejeon, 301-747, Republic of Korea \\ mhpark@cnu.ac.kr,mhgang@cnu.ac.kr
}

\begin{abstract}
In healthcare settings, interactive multimedia education using web technology is very beneficial area of education. With the rapid globalization of healthcare market, multicultural healthcare is one of the major issues that the healthcare industry is facing at this moment. The purpose of this study was to describe a research that the aim is to develop an interactive multimedia education program utilizing web interface to enhance cultural competency of the healthcare professionals. The system is being developed using the following five processes: 1) Analysis stage 2) Designing stage 3) Content framing and development stage 4) Program application stage 5) Evaluation stage. Interactive learning activities consisted of online lecture \& interactive case scenario, quiz, video \& audio, discussion, individual and team project, and $Q \& A$ to facilitate the cognitive and emotional interactive learning of the participants. The completed program was applied to nurses in the community hospitals and interactive web based multimedia education was found to be an effective e-learning media of healthcare professionals in the information age.
\end{abstract}

Keywords: e-learning, Healthcare, Culture

\section{Introduction}

\subsection{Background}

After the first web based medical education program reported in early 90's, computer assisted education has been implemented constantly. With the rapid change in working environments, there has been an increasing need of training and retraining healthcare professionals in terms of new concept, issues, technology, and services. Web based interactive multimedia learning is an important method that can be used to supplement or replace the off-line education by creating a learning environment with easy and fast access to learning experience [1].

\section{*Corresponding author}

This study was financially supported by research fund of Chungnam National University in 2012. 
Especially, e-learning for healthcare professional is one area that could benefit from the use of interactive multimedia based instruction to attract the users who have difficulties in finding time and place to learn new knowledge. Web based multimedia instruction allows the user to have control over the time and contents of their education program [2]. This ensures that the learners can study at their own pace with access to individualized study material. A variety of entertaining multimedia formats in Web based program including online lectures, video \& audio materials can attract users' interest. Furthermore, interactive discussion with peers and lecturers establishes interactive learning environments [1].

The entire healthcare market is globalizing very fast. The internet and the development of information technologies revitalized the exchanges of information and knowledge worldwide. Korea is one of the fastest globalizing countries showing significant changes in ratio of foreigners residing in Korea. Having ever increasing inflow of foreign workers and the international marriage rate, now the proportion of multicultural families is on the rise causing unprecedented social issues. Healthcare system therefore needs to be changing and evolving with the cultural norms, beliefs and values, social structure and environmental conditions. Thus, multicultural education is needed urgently to improve the cultural competency of healthcare in Korea [2].

Education on healthcare professional is typically delivered group based with text based information. The traditional education approach did not allow flexibility in learning that could act as a barrier of busy working health professional [3]. E-learning modules have become a solution that assures health professionals of access to clinical knowledge with up-to dated learning materials. This method is a realistic alternative to face-to-face teaching method that students are also known to prefer to study with [4].

There are a number of disadvantages that involve learner factors. Students have no opportunity to discuss the class with their peers that facilitates in-depth understanding of areas of teaching. In addition, students may skip difficult areas resulting in missing important emphases [5].

A high improvement of knowledge can be expected as individualized web education and multimedia can save the healthcare professional's time and efforts than offline based group education alone. Interactive multimedia can be effective tool designed to be used in tailored education, where participants can learn about specific topics in efficient ways at their workplace or home [6]. Further work by other researchers explored the effectiveness of online education, concluded that the e-learning could be an effective, efficient and convenient way to educate large numbers of users making contribution in transferring learning into practice for health professional in particular [7].

\subsection{Purpose of the Study}

In this study, we developed a web based interactive multimedia education program using user friendly interface for multicultural education program targeting the nurses in hospital settings.

The specific purposes of the study were as follows;

1) To analyze the target users' needs for education 
2) To design the system architecture and database

3) To develop e-learning education system

\section{Method}

The development of the program involved the coordinated actions of the researcher as a content expert, a web designer and database programmer for website, and a multimedia designer responsible for interface. An adaptation of the model for developing interactive multimedia was used to guide program construction. The whole process of system development is shown in Figure 1.

During the analysis stage, a group of nurses in the community hospitals was surveyed to assess the health professional's needs for education program. Previous research studies were also reviewed in terms of the learners' needs on interactive multimedia for multicultural education was investigated. Multimedia education programs, teaching materials, and websites related to multicultural education or e-learning for healthcare professionals were examined for content analysis.

Based on the result of the analysis against content, technical, and environmental need, the following topics were selected to be developed using interactive multimedia: cultural concepts, cultural awareness, history of cultural nursing, cultural beliefs of healthcare professionals, cultural communication, culture sensitive care related to gender, religion, family, birth, and death, and global healthcare markets. The educational contents were structured based on the cultural competency models and transformative education concepts.

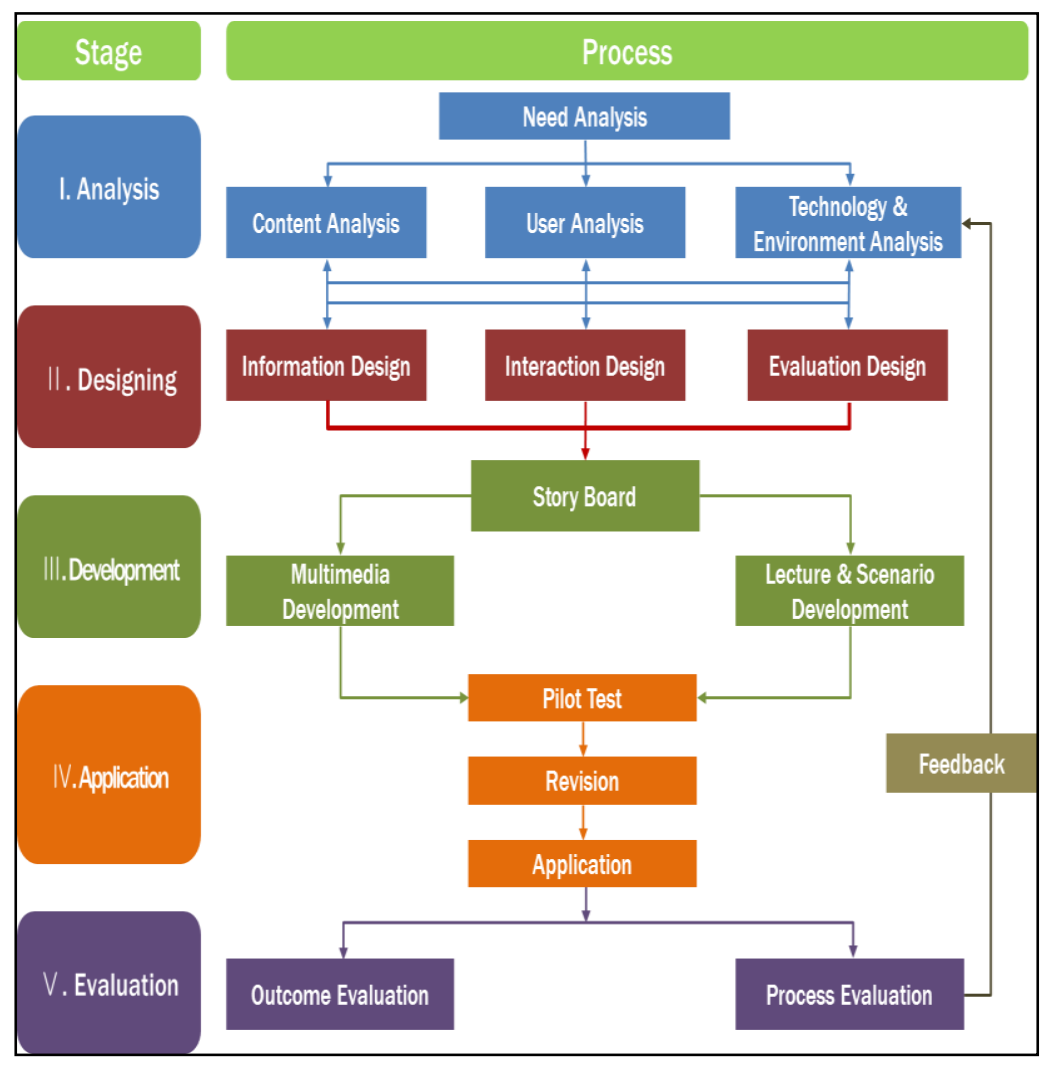

Figure 1. System Development Process 
The design phase, content was classified into texts, images, and video \& audio. Links among sites were also designed. Information of multicultural competency concepts were designed mainly using online lectures and interactive case scenarios. The main screen was designed to show the site map and to provide accesses to interactive learning, discussions, bulletin boards, and links. Post-learning discussion and Q \& A were designed to solicit users' impressions of the learning experience and facilitate interface. For motivation design, preview of each learning activity was provided in order to stimulate users' interests in learning activity. Functions to evaluate users' learning progress were designed including quizzes, online test, assignment, discussion, and activity participation check.

In the production phase, the structure of the program was organized into a sequence of modules and user friendliness considering difficulties of content, and the technological possibility of implementation. The program was developed in Windows environment using HTML, JavaScript, Flash action, photoshop, and so on (Figure 2).

The system environment of the web program was as follows;

- Server Hardware: CPU Intel E5410 QC 2.33GHz, RAM 4GB, SAS 146GB

- Server: Linux(CentOS) and provided WWW and FTP service

- Database system: MySQL(Structured Query Language) 5.0.77, Management DB and Client DB

- Master and user web programing: PHP(Personal Hypertext Preprocessor)

- PHP program development software: EditPlus Text Editor v3.21

- Graphic authoring: Adobe Flash CS5

- Web coding and html development: Adobe Dreamweaver CS5

- Web image editing: Adobe Photoshop CS5 \& Adobe Illustrator CS5 


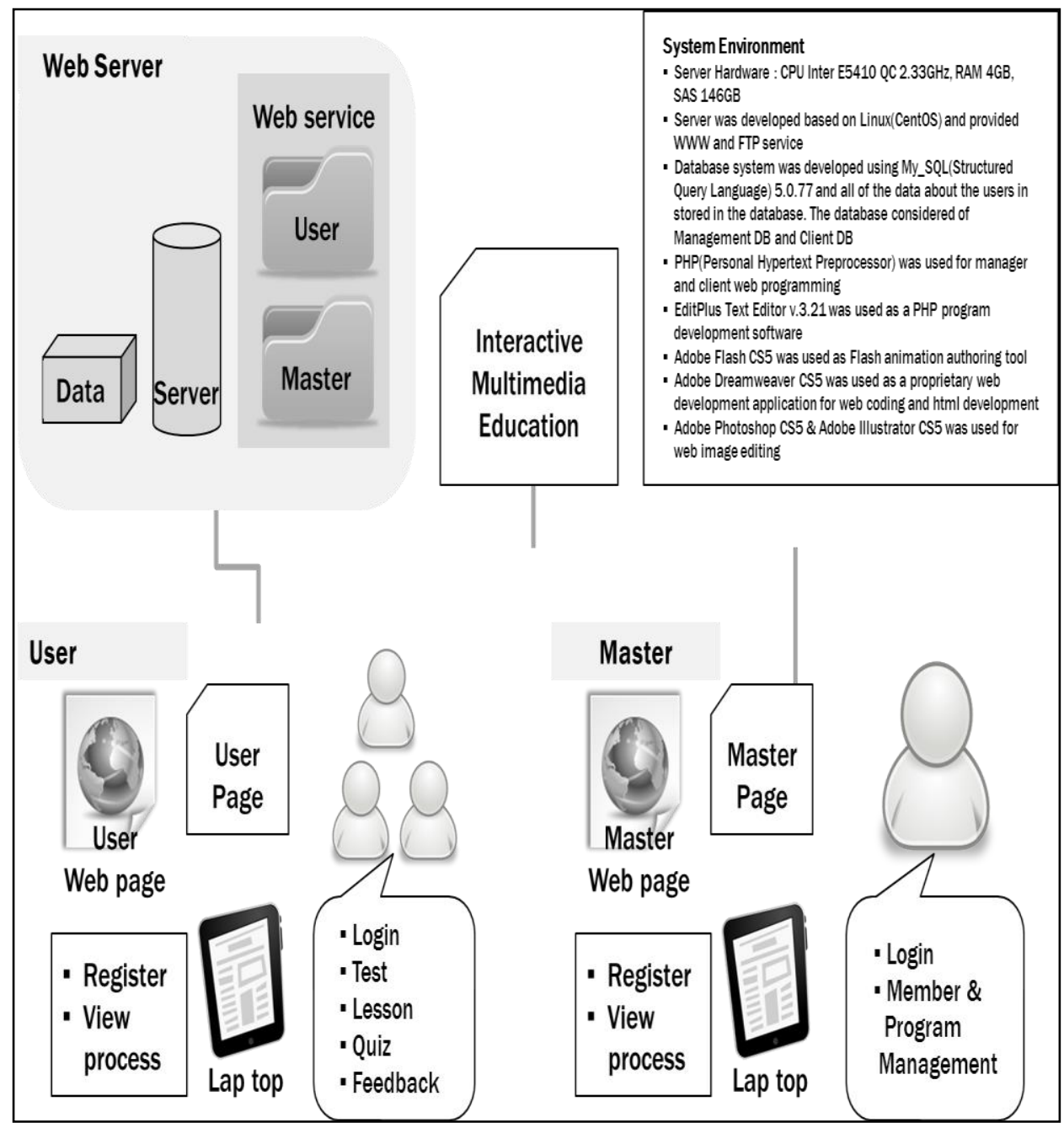

Figure 2. Structure of System

In the operation phase, produced content was uploaded on the web. Learners had the login process in the main page and needed to be approved by the instructor to use the site and then could access lessons and activities.

During the evaluative phase, the evaluation survey assessed the users' perception of the learning environment. Specifically, it consisted of 14 statements, with seven statements for each of the two categories: instructional values and instructional function. Users were required to respond to each statement a five point Likert-type scale where 5 equals strongly agree and 1 equals strongly disagree. 


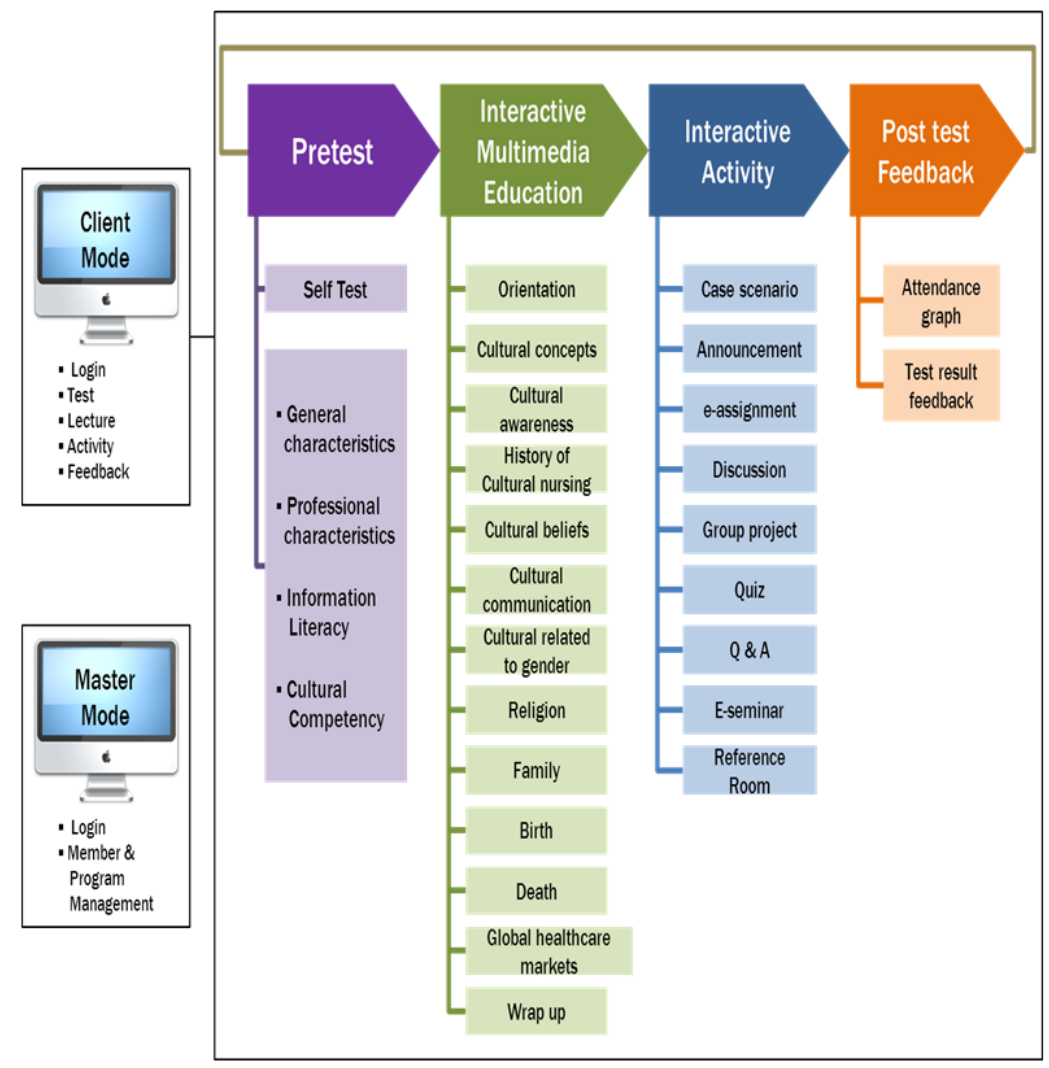

Figure 3. Design of Web-based Interactive Education

The education was guided with a main homepage which showed the route of educational sessions on the web. Each session was educated with video lecture modules which contained the information about multicultural education. All information was provided with both picture and text. The interactive learning consists of 14 multimedia lessons. Within each lesson, the visuals on screen included video lectures and power point slides. They were created for this multimedia environment and were relevant to the instructional content on page. Navigation of the lessons was sequential from Lesson 1 to Lesson 14 and no lesson could be skipped. Within each lesson, the learners had the choice to advance to the next screen, replay the current screen, or go back to the previous screen. The learners were afforded as much time as they wanted to process the information on each screen.

Interactive case scenarios were provided after each lesson. The learning environment contained a number of features including (1) its size was 800 x 600 pixels; (2) a listing of all lessons, with showing which lesson was completed by the users; (3) graphical aids indicating what lesson the learner was in (4) a control panel containing a back button, a replay button, and a next button that enabled the learner to move back a single screen, replay the current screen including video lectures or move to the next screen, respectively. The main screen provides menus such as introduction, pretest, interactive multimedia lessons, quiz, discussion room, Q\&A, bulletin boards, post-test, and link (Figure 3). If a menu is clicked, the corresponding page appears. In addition, the main menu always appears on the upper bar so that the user can select a different menu easily. 


\section{Results}

The web based learning was evaluated for two attributes, i.e., instructional value and instructional function (Figure 4). The results from users indicated high satisfaction of the participants and good quality of the web-based learning medium.
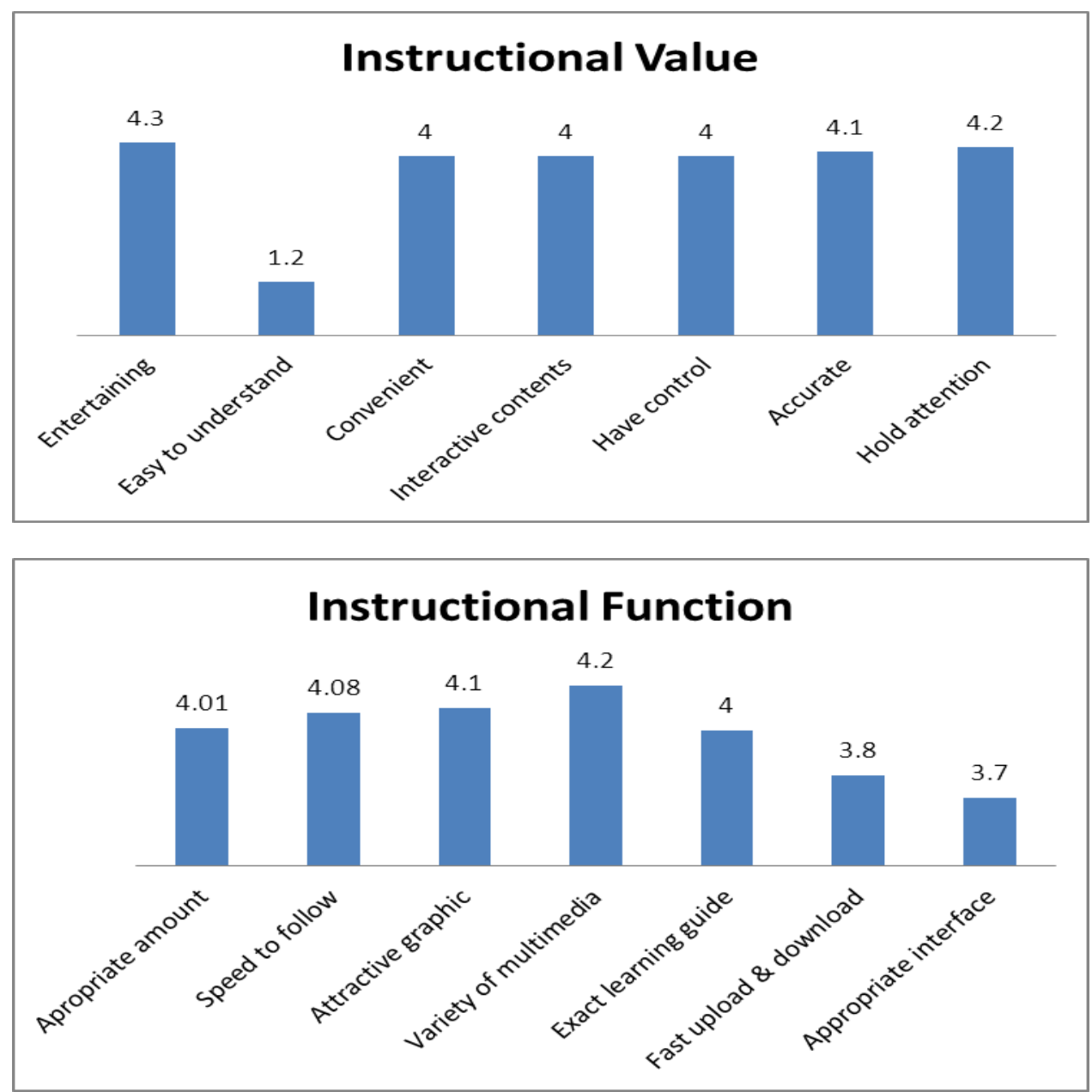

Figure 4. User's satisfaction of the system

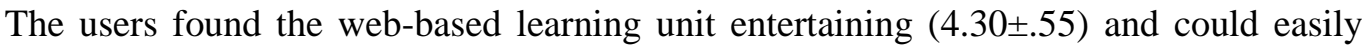

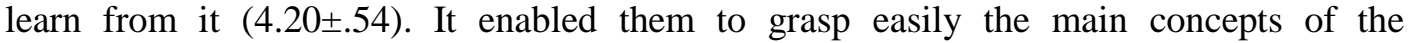
multicultural healthcare $(4.00 \pm .42)$. Interactive multimedia and interactive case scenario could effectively demonstrate the situation and context of multicultural healthcare by transforming the abstract content into concrete practice situation (4.00土.69). Additionally, quizzes and feedback assisted them to review the content and to assess themselves for knowledge development. The results also suggested that the participants perceived the

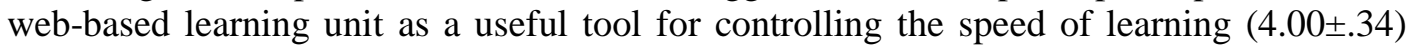
and holding attention (4.20 \pm .23$)$.

Regarding web-based design, most users indicated that the developed unit was 


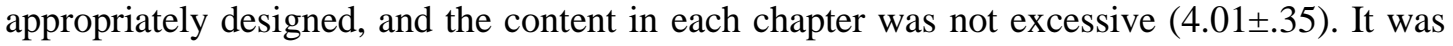
easy to navigate and follow (4.08 \pm .38$)$. The features in terms of voice, font, color, animation,

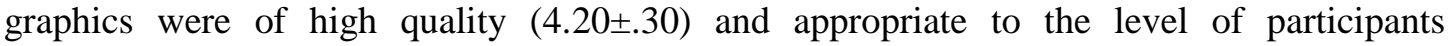

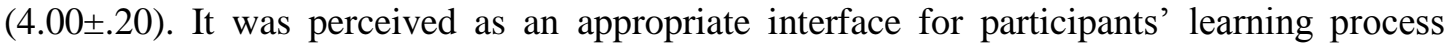
$(4.01 \pm .45)$.

\section{Discussion}

This study described the development and evaluation process of e-learning system using an interactive multimedia such as online lecture and quizzes to facilitate healthcare professionals' participation and comprehension of multicultural healthcare education. Distance learning is an opportunity for nurse educators and institutions to develop a learning environment in order to enhance continuing professional development [8]. It provides options for lifelong learning for healthcare professionals-including those working in community settings-that is effective and cost efficient. Development of web based professional development programs can contribute to widening the participation of community healthcare professionals in lifelong learning, practice and role development [9].

Previous study performed an e-learning program for cultural diversity indicated that convenience of being able to choose the time to study and being able to repeat sections without consideration of other students' need are the commonly reported preferences by students. In this previous study, however, the students would use the e-learning program partially as part of self-directed learning. Some said that they would actively participate in the program only when it is obligatory [10]. After evaluating 35 papers, a previous literature review identified four domains including knowledge gains, learner attitudes, learning efficiency and program cost. The study concluded that although the e-learning method was given high satisfaction ratings by majority of learners, this method cannot replace traditional method that involves small-group discussion or problem-based learning. Students learned more efficiently with provision of well-designed curriculum and study materials. It is therefore recognized by educator that program should be tailored to learner's needs [11]. These reflect potential problems associated with e-learning program which requires additional method to facilitate effective e-learning environment.

This study has also shown that the web based e-learning education can be successful in communicating continuing education information such as multicultural education, particularly if they are developed in collaboration with the target population. Previous studies recommended that web based multimedia education needed to be developed based on the target user's culture level and learning style. In this study, the researcher developed the learning modules while working with the focus group of nurses in a formative manner, during the development phase [12]. In this study, multimedia lectures with interactive case scenario were used. Augmentation of self-directed learning via an interactive multimedia can provide the users with the significant advantage of increased knowledge and skills. Interactivity, practice exercises, repetition, and feedback seem to be associated with improved learning outcomes. This may be due to the interactive feature of the unit with animation, colorful pictures, interactive quizzes, exercises and case scenarios [13].

\section{Conclusion}

The web-based individualized interactive education is an innovative approach that provides a mean for healthcare workers to improve their competence for new technology and knowledge. It empowers nurses with different knowledge levels to enhance their competence for multicultural healthcare. The results from satisfaction survey revealed that the students 
perceived the web-based learning unit to be fun, flexible, and convenient.

This paper considers the opportunities that web-based and online education programs can provide healthcare professionals to promote professional skills while maintaining a work-life balance, and the role of the lecturer in successfully supporting professionals on web-based learning programs. Development of e-learning program takes significant effort in designing and delivering of a program. Not only cost and time of development, but also preference and convenience of learner should be taken into consideration as attitude of learner handles the success of program.

It is needed to conduct a study in various environments by expanding the range of subjects and subject environment of this multicultural education program. In addition, there is a limitation in the interpretation of the results of study in this study because this study checked the applicability of education with a group of nurses of a night special entry course of a college as the subject, and thus web based multicultural education programs should be developed in various methods and be expanded to an education with health care professionals actually working and students as the subject.

\section{Acknowledgements}

This study was financially supported by research fund of Chungnam National University in 2012.

\section{References}

[1] L. Howatson-Jones, "Designing web-based education courses for nurses", Nursing Standard, vol. 19, no. 11, (2004), pp. 41-44.

[2] D. A. Boyle, A. Sheridan, J. McClary and J. White, "A multifocal education strategy to enhance hospitalbased cultural competency in professional staff”, Oncology Nursing Society, vol. 29, no. 5, (2002), pp. 764768.

[3] G. R. Westmoreland, S. R. Counsell, W. Tu, J. Wu and D. K. Litzelman, "Web-Based Training in Geriatrics for Medical Residents: A Randomized controlled trial using standardized patients to assess outcomes", Journal of American Geriatric Society, vol. 58, (2010), pp. 1163-1169.

[4] R. M. Harden and I. R. Hart, "A new vision for distance learning and continuing medical education". Journal of Continuing Education in the Health Professions, vol. 25, (2005), pp. 43-51.

[5] P. Vivekananda-Schmidt, M. Lewis and A. B. Hassell, "Cluster randomised controlled trial of the impact of a computer-assisted learning package on the learning of musculoskeletal examination skills by undergraduate medical students", Arthritis Rheum, vol. 53, (2005), pp. 764-771.

[6] D. A. Cook, A. J. Levinson, S. Garside, D. M. Dupras, P. J. Erwin and V. M. Montori, "Instructional design variations in internet-based learning for health professions education: A systematic review and meta-analysis", Academic Medicine, vol. 85, no. 5, (2010), pp. 909-922.

[7] T. Wang, "Web-based dynamic assessment: Taking assessment as teaching and learning strategy for improving students' e-Learning effectiveness", Computers \& Education, vol. 54, (2010), pp. 1157-1166.

[8] D. M. Billings, H. R. Connors and D. J. Skiba, "Benchmarking Best Practices in Web-Based Nursing Courses", Advances in Nursing Science, vol. 23, no. 3, (2001), pp. 41-52.

[9] T. R. Crawford, "Using problem-based learning in web-based components of nurse education". Nursing Education in Practice, vol. 11, no. 2, (2011), pp. 124-130.

[10] K. Hawthorne, H. Prout, P. Kinnersley and H. Houston, Evaluation of different delivery modes of an interactive e-learning programme for teaching cultural diversity. Patient Education and Counseling, vol. 74, no. 1, (2009), pp. 5-11.

[11] H. S. Chumley-Jones, A. Dobbie and C. L. Alford, "Web-based learning: sound educational method or hype? A review of the evaluation literature", Academic Medicine, vol. 77, no. 10, (2002). pp. S86-S93.

[12] M. Jou and Y. S. Wu, "Development of a web-based system to support self-directed learning of microfabrication technologies", Educational Technology \& Society, vol. 15, no. 4, (2012), pp. 205-213.

[13] J. Southernwood, "Distance learning-the future of continuing professional development", Community Practitioners, vol. 81, no. 10, (2008), pp. 21-23. 


\section{Authors}

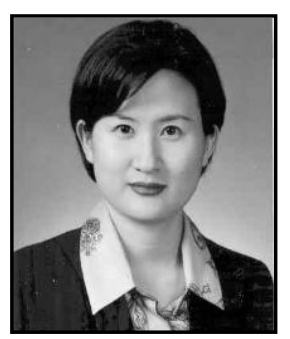

\section{Myonghwa Park}

She is an associate professor at college of nursing, Chungnam National University. Her research area includes e-learning, health informatics, and evidence based nursing.

\section{Moonhee Gang}

She is an assistant professor at college of nursing, Chungnam National University. Her research area includes nursing education and psychiatric nursing. 